\title{
Laryngopharyngeal reflux induced sleep-related laryngospasm
}

\author{
Ross $\mathrm{O}^{\prime}$ Shea ${ }^{1}\left[\right.$ : Máire Gaffney ${ }^{1} \cdot$ Majura Kaare $^{2} \cdot$ John Eugene Fenton ${ }^{2}$
}

Received: 12 December 2021 / Accepted: 19 January 2022 / Published online: 31 January 2022

(c) The Author(s) 2022

\begin{abstract}
Background Sleep-related laryngospasm (SRL) has been defined as the sustained closure of the vocal cords during sleep. Studies have suggested that it is a rare manifestation of laryngopharyngeal reflux (LPR). Difficulties in diagnosing SRL and LPR have led to the condition being under-recognised in the clinical setting.

Aims The aim of this study was to determine if LPR was the cause of the SRL symptoms seen in our patients.

Methods A retrospective chart assessment of patients with SRL. Patients with risk factors for LPR were identified. These included smoking status, alcohol intake, a history of dyspepsia or history of gastroesophageal reflux disease, a history of late-night eating and a history of eating spicy or fatty foods before bed. A clinical diagnosis based on the history and response to management was made for the diagnosis of LPR. All were advised to refrain from late meals and those with signs of nasopharyngitis were commenced on proton pump inhibitor therapy.

Results Nineteen patients (mean age \pm SD: $57.21 \pm 15.18$ ) were included in the study. All had at least one risk factor for LPR. Ten $(52.6 \%)$ had signs of nasopharyngitis on nasendoscopy. Following treatment, 17 (89.5\%) reported no further SRL symptoms at 1-year follow-up.

Conclusion SRL is a largely unknown and under-diagnosed condition. We believe this study provides supportive evidence for the causal relationship between LPR and SRL.
\end{abstract}

Keywords Diagnosis $<$ General Gastro-oesophageal reflux $<$ Pharynx $\cdot$ Larynx - Obstructive sleep apnoea $<$ Rhinology . Sleep medicine $<$ General $\cdot$ Stridor $<$ Larynx

\section{Introduction}

Laryngospasm has been defined as the sustained closure of the vocal cords which results in partial or complete loss of airway patency [1]. Sleep-related laryngospasm (SRL) exclusively occurs while the patient is asleep and is characterised by sudden awakening with an inability to breathe. These episodes last seconds and can also be associated with stridor [2]. Patients present clinically well. They report episodes of acute choking attacks that occur at night and are short lasting. Clinical features and polysomnographic studies have previously suggested SRL to be a spasm of the vocal

Ross O'Shea

Rossoshea95@gmail.com

1 Faculty of Education and Health Sciences, University of Limerick Medical School, Garraun, Castletroy, Limerick, Ireland

2 Department of Otorhinolaryngology Head and Neck Surgery, University Limerick Medical School, Limerick, Ireland cords of unknown aetiology [3]. Fear of suffocation while sleeping has also been reported which has a detrimental effect on the patient's quality of life [2]. Despite this, SRL is a condition not well described in the literature.

The term "sleep-related laryngospasm" was first described in a case series by Aloe and Thorpy which suggested laryngopharyngeal reflux (LPR) was a possible explanation for SRL [3]. LPR refers to the acute or chronic backflow of gastric contents of liquid or aerosol, into the larynx and/or pharynx and is considered to be relatively common in the general population. Although termed "silent reflux" in the past, it can present with a multitude of complaints involving the upper aerodigestive tract including an association with globus pharyngeus, chronic rhinosinusitis, a sore throat, dysphagia, chronic cough, and hoarseness [4]. SRL is believed to be a rare but severe manifestation of LPR [2, 5]. Lower oesophageal sphincter dysfunction is likely to be the probable cause of LPR. Risk factors for LPR include late meals, spicy or fatty foods, stress, cigarette smoking, caffeine intake and alcohol consumption $[4,6]$. The incidence 
of SRL is still unknown but previous studies have estimated that $10-30 \%$ of patients that seek ENT care, present with symptoms of LPR [7].

Differential diagnoses for SRL include obstructive sleep apnoea (OSA), nocturnal asthma, sleep terror episodes and seizures. These need to be excluded in order for an accurate diagnosis of SRL to be made. OSA is the most common sleep-related breathing disorder and therefore must be ruled out before diagnosing SRL. Patients with OSA will not generally awaken themselves and will not report stridor nor difficulty breathing when awake. If such symptoms are present, then a likely diagnosis of SRL can be made; however, if the history is suggestive of OSA or nocturnal asthma, then the patient should be referred for a respiratory assessment.

LPR is notoriously difficult to diagnose and remains a controversial condition. Definitive criteria have not been universally agreed although multiple suggestions have been proffered. Diagnosis and management vary significantly from country to country [8]. LPR scoring systems based on the history and findings include the reflux finding score (RFS), reflux symptom index (RSI), reflux sign assessment (RSA), Horvath score and Sataloff score, which are cumbersome to perform in a busy clinic [7-10]. None of these scoring systems have included SRL as a factor. A previous study has demonstrated a relationship between sleep-related gastroesophageal reflux and SRL through the use of overnight polysomnogram and $\mathrm{pH}$ monitoring; however, these investigations are not readily available in general otorhinolaryngology - head and neck surgery practices [2]. Additionally, the most accurate placement of the $\mathrm{pH}$ probe has not yet been agreed upon. Despite all of these diagnostic tools, the nasopharynx is historically excluded on clinical investigation in patients with LPR while endoscopic subtleties in the epipharyngeal mucosa may have been missed in the few papers that have addressed the postnasal space in their studies [11-13]. These studies did, however, observe areas of the postnasal space to be highly reliable in response to change. We believe isolated nasopharyngitis is an important finding indicating the presence of LPR. Evidence of upper respiratory inflammation such as rhinosinusitis whether it be acute or chronic would suggest a cause other than LPR. The senior author (JEF) has developed an approach in an iterative manner for diagnosis and management during his 30 years of clinical experience. This evolving method combines a comprehensive history with findings of any degree of nasopharyngitis and/or posterior commissure oedema for a presumptive diagnosis of LPR. The confirmation of the diagnosis is an improvement in symptoms and a reduction or resolution of inflammatory findings following a 3-month course of twice daily low-dose PPI, tapering to a single evening dose for 1 month. This empiric treatment protocol has been adopted by some authors and will be used for the presumptive diagnosis of LPR in this study [14-16]. The aim of our study is to investigate if a direct association between LPR and SRL exists, suggesting LPR is a causative factor in the aetiology of SRL in our patients. We hope to present arguments supporting this conclusion.

\section{Materials and methods}

\section{Ethical consideration and study design}

The Research Ethics Committee of the University Hospital Limerick granted formal approval (Ref 148/2020). A retrospective chart assessment was carried out involving consecutive patient's that presented to the routine outpatient's service of the senior author.

\section{Study population}

The study was conducted in an academic tertiary care medical centre. Inclusion criteria included all patients with a history of nocturnal choking attacks. Differential diagnoses such as OSA, nocturnal asthma, sleep terror episodes and seizures were deemed unlikely through careful history and examination. Patients with a history or clinical findings suggestive of any of these differentials were excluded from the study. Patients with suspected OSA or nocturnal asthma were referred to respiratory for further investigations. Patients with a history of nocturnal choking attacks suggestive of SRL subsequently underwent flexible nasopharyngoscopy.

\section{Methods}

Patient charts during a 5-year period from 2016 to 2020 inclusive were examined for patients that presented with or were found on routine history-taking to have symptoms suggestive of SRL. Reflux risk factors for reflux were also recorded. These included smoking status, alcohol intake, a history of dyspepsia or history of gastroesophageal reflux disease, a history of late-night eating and a history of eating spicy or fatty foods before bed. Patients were classified as having risk factors for LPR if any of these criteria were present. Episodes of nocturnal choking were recorded as a presenting symptom or as discovered on direct questioning by including the enquiry as part of a standard history in patients with suspected LPR or sleep disordered breathing.

All patients underwent nasopharyngoscopy by a single consultant otolaryngologist. The presence of nasopharyngitis on endoscopy was noted. Other abnormalities associated with reflux including posterior commissure oedema and hypertrophy were also noted. Criteria for severity of nasopharyngitis are defined in Table 1. Patients with risk factors for LPR or signs of nasopharyngitis on endoscopy were classified as having suspected LPR. 
Table 1 Nasopharyngitis severity

\begin{tabular}{ll}
\hline Severity & Characteristic \\
\hline Mild & Minimal erythema with subtle line of demarcation [11, \\
& $12]$ \\
Moderate & Granular or raised mucosal erythematous changes \\
Severe & Crusting of the mucosa or blood-stained mucosa \\
\hline
\end{tabular}

All patients with risk factors for LPR were advised to refrain from eating less than $2 \mathrm{~h}$ before bed and to avoid the eating of spicy or fatty foods. Smoking cessation and a reduction in alcohol intake were also recommended if applicable to all patients. Patients without nasopharyngitis on nasendoscopy were advised to take a trial of Gaviscon for 6-8 weeks in addition to lifestyle changes. Patients with signs of nasopharyngitis on nasendoscopy were initiated on a $20 \mathrm{mg}$ twice daily proton pump inhibitor (PPI) for 3 months which was taken $30 \mathrm{~min}$ to $1 \mathrm{~h}$ before meals. The evening dose was prescribed for a month to prevent rebound symptoms with omission of the morning dose after the initial month. Gavison at night and lifestyle changes were also recommended.

Patients were asked to record any further symptoms or reoccurrences of their nocturnal choking attacks. All patients were monitored remotely by telephone if symptoms failed to settle. Patients with signs of nasopharyngitis on endoscopy were followed up at 4 months with repeat nasopharyngoscopy following PPI therapy.

\section{Statistical analysis}

All statistical analyses were performed using the Statistical Package for the Social Science (SPSS) version 25 software (SPSS Inc., Chicago IL).

\section{Results and analysis}

\section{Patient demographics}

Nineteen patients (mean age \pm SD: $57.21 \pm 15.18$ ) were included in the study. Fifty-eight percent were female, 10.5\% were current smokers and $100 \%$ admitted to alcohol intake. None of our patients had a history of epilepsy or seizures and none had a history of anxiety or panic attacks.

\section{Risk factors for LPR and nasopharyngitis on nasendoscopy}

Thirteen patients (68.4\%) had a history of dyspepsia or history of gastroesophageal reflux disease. Seven patients $(36.8 \%)$ had a history of late-night meals or admitted to the eating of spicy or fatty foods less than $2 \mathrm{~h}$ before bed. All 19 patients had at least one risk factor for LPR. Eight patients (42.1\%) had two risk factors for LPR, and 7 patients (36.8\%) had three risk factors. Ten patients $(52.6 \%)$ had signs of nasopharyngitis on nasendoscopy. There was no correlation between number of risk factors and flexible nasopharyngoscopy findings.

\section{Posterior commissure hypertrophy/oedema}

Seven patients (36.8\%) had signs of posterior commissure hypertrophy or oedema on nasendoscopy. Two of 7 (28.6\%) patients with posterior commissure hypertrophy did not have signs of nasopharyngitis on nasendoscopy.

\section{Presenting complaint}

Thirteen of the 19 patients presented with nocturnal choking attacks. Number of choking attacks ranged from 1 to 4 attacks per year. Choking attacks lasted less than $10 \mathrm{~s}$ in all instances based on patient history and collateral history from partner if available. Six patients (31.5\%) did not volunteer episodes at presentation but admitted to nocturnal choking attacks after direct questioning during history taking.

\section{Symptom reoccurrence}

Two of 19 patients (10.5\%) had a reoccurrence of symptoms within 1 year of initial consultation. Both patients suffered just one attack each while confirming that a large late meal with excessive alcohol intake preceded the event.

\section{Discussion}

SRL is a condition that has been under-reported in the medical literature. It remains a diagnosis that needs to be considered and studied further to realise its true prevalence and aetiology. This study reveals that SRL is generally not a common problem but is likely to be more frequent than suspected heretofore. It appears to be an infrequent but frightening occurrence for those who have experienced it, although a previously reported series dealt with a group of patients that were more severely impacted including daytime events than any in our cohort [14].

Sudden awakening and nocturnal breathing disturbances can occur for reasons other than SRL. OSA is the most common breathing-related sleep disorder. OSA is characterised by pharyngeal narrowing which results in upper airway obstruction during sleep. These patients typically will not awaken with persistent difficulty breathing or stridor. They also are unlikely to awaken themselves during sleep. OSA patients will also usually have a history 
of loud snoring and daytime sleepiness. These features are not characteristic of SRL [14]. Patients with suspected OSA were excluded from the study and were referred for sleep studies. Nocturnal asthma is another possible cause of sudden awakening and difficulty breathing. In addition to night-time symptoms, patients with nocturnal asthma will have clinical features of asthma during the day. Symptoms such as persistent dry cough and chest tightness during the day are not typical of SRL. This information was used to exclude patients with suspected nocturnal asthma from the study. Additionally, patients with a pre-existing diagnosis of asthma were excluded from the study. It was anticipated that patients who did not respond to our management regime would require referral to the respiratory team for sleep studies or for assessment of nocturnal asthma. Patients suffering from stress or emotional anxiety can also present with features resembling SRL. Sleep terror episodes can be characterised by sudden arousal accompanied by shortness of breath and behavioural manifestations of intense fear [3]. Seizures may also cause a presentation that is like that of SRL. For this reason, they should be investigated further if suspected. It is important to distinguish between these differential diagnoses when considering SRL as a possible cause of sudden awakening with breathing disturbances. A correct diagnosis of SRL is needed to adequately treat the patient. A detailed history is required from the patient to ensure other differentials are not the cause of the patient's symptoms. A neurological and otolaryngologic examination should also be performed to identify the aetiology of SRL. All of our patients were careful screened for differential diagnoses and were only included in the study if differentials were ruled out. None of the patients included in the study had proven or suspected OSA, nocturnal asthma, sleep terrors or seizures based on the history and clinical findings.

This study shows a probable association between LPR and SRL. Acute or chronic LPR is likely to be present in all our patients with suspected SRL. We have identified two potential groups. One cohort has no history suggestive of LPR with no evidence of upper aerodigestive tract inflammation and their reflux event is entirely related to a late meal while the second set have ongoing LPR with an exacerbation due to eating later than recommended. All patients had at least one risk factor for LPR while patients with two or three risk factors represented $42 \%$ and $37 \%$, respectively. These risk factors can bring about acute episodes of LPR. We hypothesise that acute LPR is the cause of the SRL symptoms seen in our patients who did not have any signs of nasopharyngitis on endoscopy. We believe the management of SRL in these patients should include the eating of food no less than $2 \mathrm{~h}$ before bed and to avoid the ingestion of spicy or fatty foods. Alcohol consumption should also be limited and smoking ideally should be ceased.
Nasopharyngitis or epipharyngitis has become more obvious with the advent of the nasopharyngoscope and for those clinicians who specifically look for it during endoscopy. It can be a subtle finding that is easily missed but the line of demarcation as described by Neri et al. does appear to be a clinical indicator of note [12]. The results in our series also show that $53 \%$ of patients had signs of nasopharyngitis on nasendoscopy. Nasopharyngitis is believed to occur following exposure of the nasopharynx to chronic LPR. We agree with a recent work that suggested a nasopharyngeal probe be used to assess duration and frequency of reflux events in future studies [10]. In patients with signs of nasopharyngitis on nasendoscopy, we suggest the introduction of a twice daily PPI in addition to the changing of eating habits, Gaviscon prior to bed if there has been a late meal, a limit to alcohol intake and the cessation of smoking. A 3-month course of twice daily low-dose PPI tapering to a single evening dose for 1 month is recommended as per previous authors [15-18].

At 1-year follow-up, 17 patients (89.5\%) reported no further SRL symptoms. The two male patients that reported symptoms, did so at Christmas time following a large late meal and the ingestion of excessive amounts of alcohol. Both patients reported only one reoccurrence of symptoms. This suggests that our treatment approach has helped to reduce the frequency of the events in our cohort. It is noteworthy that the majority of our SRL patients were female and presented between the ages of 50 and 70 . This is consistent with previous studies on SRL and LPR but contradicts the original paper $[2,14,19]$. This may be due to the relatively rare and fleeting events reported by our patients and that females do tend to seek a medical opinion earlier than males [20,21]. It may also reflect the mistaken opinion that the event was part of a gasp in patients with sleep apnoea.

The reflux finding score (RFS), published by Belafsky et al. in 2002, considers the presence of posterior commissure hypertrophy in the diagnosis or LPR. Posterior commissure hypertrophy was visualised in seven of our patients. However, the RFS was not used in this study as it does not include the nasopharynx in the diagnosis of LPR [22]. Neither was the reflux symptom index (RSI) used in this study as Chen et al. concluded that no linear relationship existed between RFS and RSI, while they also found asymptomatic patients could present with relatively high RFS scores [23]. Although coughing after eating or after lying down and breathing difficulties or choking episodes are included as two of the nine symptoms in the RSI, patients with LPR are considered usually upright refluxers and SRL has not been described in this outcome instrument [22]. Neither has SRL been alluded to in other more recently published scoring systems or LPR reviews [7-10].

We feel that the nasopharynx should be scrutinised more carefully and that this could have altered the significance of 
nasopharyngitis and the RSA score in the study by Lechien et al. [13]. In an era where patient reported outcome measures (PROMs) are topical, the ability to listen to the patient and clinical experience are paramount. We would like to highlight the importance of history taking in the diagnosis of SRL. A proportion of our population (31.5\%) did not volunteer symptoms of SRL and only admitted to nocturnal choking attacks after direct questioning. SRL is a condition that appears to occur sporadically and can be easily overlooked by patients and physicians during history taking. It is our belief that SRL is largely under-diagnosed for this reason. It is our recommendation that all patients with a suspected history of LPR or sleep-disordered breathing should be screened for SRL by asking about nocturnal choking attacks. Attention to detail in listening to the patient will also help to differentiate the episodes of SRL from the more common sleep disturbance in sleep apnoea.

Although there is no evidence-base for SRL nor for nasopharyngitis as a sign of LPR, patients with a worrying complaint were managed successfully with relatively easy and straightforward solutions. Limitations to this study include its small sample size and the lack of a definitive diagnostic test. Currently, the diagnosis depends on a combination of factors including the description of symptoms, the elimination of other potential causes, the presence or absence or nasopharyngitis, and the response to empiric management. Due to the prevalence of SRL, a small sample size is an issue that is hard to overcome in a single tertiary care medical centre. We would suggest future studies include multiple sites for a better representation of the population. Additionally, future studies would ideally include the use of overnight polysomnogram and $\mathrm{pH}$ monitoring as has been demonstrated in previous studies [2]. However, our recommendation would be, if possible, to place the upper $\mathrm{pH}$ probe in the nasopharynx coupling with a hypopharyngeal sensor. Despite the limitations, this study produced results suggesting that a relationship between SRL and LPR exists. The extent of this relationship requires additional investigation and the inclusion of a control group. Future studies should focus on increasing the study population to further investigate the treatment of SRL through the management of LPR. The response of nasopharyngitis to empiric PPI treatment for LPR is currently the subject of an ongoing retrospective study at our department.

\section{Conclusion}

SRL is a largely unknown and under-diagnosed condition. Additional research into the true aetiology of SRL is required to successfully treat the disorder. We believe this study provides supportive evidence for the causal relationship between LPR and SRL. As such, it would suggest that
SRL can and should be treated by limiting the risk of LPR. We also recommend the screening of all patients with a history of reflux for SRL as symptoms are often not volunteered during the history.

Author contribution All authors contributed to the study conception and design. Material preparation, data collection and analysis were performed by Ross O'Shea, Máire Gaffney and Majura Kaare. The first draft of the manuscript was written by Ross O'Shea and all authors commented on previous versions of the manuscript. Writing, reviewing and editing were performed by Ross O'Shea and John Eugene Fenton. Study was performed under the supervision of John Eugene Fenton.

Funding Open Access funding provided by the IReL Consortium

\section{Declarations}

Ethics approval Ethical approval was granted by the Research Ethics Committee at University Hospital Limerick (Ref 148/2020). All procedures performed were part of routine patient care.

Consent to participate Informed consent was obtained from all individual participants included in the study.

Consent to publication Informed consent to publish was obtained from all individual participants included in the study.

Conflict of interest The authors declare no competing interests.

Open Access This article is licensed under a Creative Commons Attribution 4.0 International License, which permits use, sharing, adaptation, distribution and reproduction in any medium or format, as long as you give appropriate credit to the original author(s) and the source, provide a link to the Creative Commons licence, and indicate if changes were made. The images or other third party material in this article are included in the article's Creative Commons licence, unless indicated otherwise in a credit line to the material. If material is not included in the article's Creative Commons licence and your intended use is not permitted by statutory regulation or exceeds the permitted use, you will need to obtain permission directly from the copyright holder. To view a copy of this licence, visit http://creativecommons.org/licenses/by/4.0/.

\section{References}

1. Gavel G, Walker R (2014) Laryngospasm in anaesthesia. Cont Educ Anaesth Crit Care Pain 14:47-51

2. Roland MM, Baran AS, Richert AC (2008) Sleep-related laryngospasm caused by gastroesophageal reflux. Sleep Med 9:451-453

3. Aloe FS, Thorpy MJ (1995) Sleep-related laryngospasm. Arq Neuropsiquiatr 53:46-52

4. Postma GN, Halum SL (2020) Laryngeal and pharyngeal complications of gastroesophageal reflux disease. https://www.nature. com/gimo/contents/pt1/full/gimo46.html. Accessed 24 Nov 2020

5. Iriarte J, Urrestarazu E, Alegre Met al (2006) Sleep-related laryngospasm: a video-polysomnographic recording. Epileptic Disord 8:70-72

6. Massawe WA, Nkya A, Abraham ZS et al (2020) Laryngopharyngeal reflux disease, prevalence, and clinical characteristics in ENT department of a tertiary hospital Tanzania. World J Otorhinolaryngol Head Neck Surg 7:28-33 
7. Lechien JR, Mouawad F, Bobin F et al (2021) Review of management of laryngopharyngeal reflux disease. Eur Ann Otorhinolaryngol Head Neck Dis 138:257-267

8. Lechien JR, Allen JE, Barillari MR et al (2021) Management of laryngopharyngeal reflux around the world: an international study. Laryngoscope 131:1589-1597

9. Horvath L, Hagmann P, Burri E et al (2021) A novel scoring system for evaluating laryngopharyngeal reflux. Clin Otolaryngol 46:594-601

10. Vance D, Park J, Alnouri G et al (2021) Diagnosing laryngopharyngeal reflux: a comparison between 24-hour $\mathrm{pH}$-impedance testing and pharyngeal probe (Restech) testing, with introduction of the Sataloff Score [published online ahead of print, 2021 Jun 4]. J Voice S0892-1997(21)00136-3

11. Mogitate M, Sasaki Y, Komiyama A (2021) Outcome of an outpatient specialty clinic for chronic epipharyngitis. Auris Nasus Larynx 48:451-456

12. Neri G, Pugliese M, Castriotta A et al (2013) White-line: a new finding in laryngopharyngeal reflux objective evaluation. Med Hypotheses 80:769-772

13. Lechien JR, Rodriguez Ruiz A, Dequanter D et al (2020) Validity and reliability of the reflux sign assessment. Ann Otol Rhinol Laryngol 129:313-325

14. Thurnheer R, Henz S, Knoblauch A (1997) Sleep-related laryngospasm. Eur Respir J 10:2084-2086

15. Spantideas N, Drosou E, Bougea A et al (2020) Proton pump inhibitors for the treatment of laryngopharyngeal reflux. A systematic review. J Voice 34:918-929

16. Wei C (2016) A meta-analysis for the role of proton pump inhibitor therapy in patients with laryngopharyngeal reflux. Eur Arch Otorhinolaryngol 273:3795-3801
17. Lee YC, Lee JS, Kim SW et al (2013) Influence of age on treatment with proton pump inhibitors in patients with laryngopharyngeal reflux disease: a prospective multicenter study. JAMA Otolaryngol Head Neck Surg 139:1291-1295

18. Orr WC, Robert JJ, Houck JR et al (2009) The effect of acid suppression on upper airway anatomy and obstruction in patients with sleep apnea and gastroesophageal reflux disease. J Clin Sleep Med $5: 330-334$

19. Lechien JR, Bobin F, Muls V et al (2021) The efficacy of a personalised treatment depending on the characteristics of reflux at multichannel intraluminal impedance-pH monitoring in patients with acid, non-acid and mixed laryngopharyngeal reflux. Clin Otolaryngol 46:602-613

20. Tudiver F, Talbot Y (1999) Why don't men seek help? Family physicians' perspectives on help-seeking behavior in men. J Fam Pract 48:47-52

21. Banks I (2001) No man's land: men, illness, and the NHS. BMJ (Clin Res Ed) 323:1058-1060

22. Belafsky PC, Postma GN, Koufman JA (2002) Validity and reliability of the reflux symptom index (RSI). J Voice 16:274-277

23. Chen M, Hou C, Chen T et al (2018) Reflux symptom index and reflux finding score in 91 asymptomatic volunteers. Acta Otolaryngol 138:659-663

Publisher's Note Springer Nature remains neutral with regard to jurisdictional claims in published maps and institutional affiliations. 